\title{
Effects of optical feedback with dispersive loss on the amplitude noise and relaxation resonance of semiconductor lasers
}

\author{
J. Kitching, R. Boyd and A. Yariv \\ Department of Applied Physics, California Institute of Technology, \\ 128-95, Pasadena, CA 91125 \\ Y. Shevy \\ Department of Physics, University of Miami, \\ CoralGables, FL 33134
}

\begin{abstract}
Optical feedback from an external cavity containing an element of dispersive loss was used to reduce the amplitude noise of a semiconductor laser. At feedback levels of $P_{f b} / P_{\text {out }} \approx 10^{-2}$, a maximum amplitude noise reduction of $16 \mathrm{~dB}$ was measured close to threshold but the potiential for reduction was reduced considerably at higher injection currents as the laser noise approached the shot noise limit. In addition, the threshold current decreased and the linewidth was reduced to $10 \mathrm{kHz}$. The relaxation oscillation peak in the amplitude noise spectrum was also found to be dramatically suppressed and we find evidence that the relaxation resonance can be moved to much higher frequencies using optical feedback techniques.
\end{abstract}

\section{INTRODUCTION}

Optical feedback has long been known to dramatically modify the noise and dynamic properties of semiconductor lasers ${ }^{1-8}$. The prospect of using optical feedback techniques to narrow the laser linewidth, reduce the amplitude noise and improve the modulation response has resulted in considerable theoretical and experimental effort to determine optimum feedback conditions. Linewidth reduction is one area in which significant strides have been made using optical feedback. Reduction of the laser linewidth by several orders of magnitude ${ }^{9-11}$ is possible using weak feedback from a strongly dispersive element such as an external FabryPerot cavity or an atomic transition. It has also been predicted that the relaxation oscillation frequency and damping rate can be significantly modified by optical feedback ${ }^{2}$.

In contrast to the situation with linewidth reduction, the effects of optical feedback on the semiconductor laser amplitude noise spectrum have received relatively little attention. Theoretical investigations have 
predicted reductions in the amplitude noise by as much as $16 \mathrm{~dB}$ over a broad frequency range ${ }^{4,12}$ but such predictions have not yet received conclusive experimental verification. Although low frequency amplitude noise reduction by as much as $12 \mathrm{~dB}$ has been observed under feedback conditions ${ }^{13,9}$, there has been no extensive experimental investigation made, to our knowledge, into the behaviour of and limits to this reduction. This is especially true for the quantum aspects of semiconductor laser amplitude noise ${ }^{14,15}$ and the possibility of producing enhanced squeezing through a combination of pump suppression and optical feedback ${ }^{16}$.

In this paper we investigate the effects of strong $\left(P_{f b} / P_{\text {out }}=10^{-2}\right)$ optical feedback on the amplitude noise spectrum of a semiconductor laser. Specifically, we examine its dependence on frequency, pump rate and feedback parameters. Finally, we present results on the effects of optical feedback on the relaxation oscillation frequency.

\section{EXPERIMENT}

The experimental setup, shown in Figure 1, has been described previously ${ }^{11}$. A single-mode GaAs semiconductor laser (STC LT50A-03U) lasing at $852 \mathrm{~nm}$ and with a threshold current of $51.4 \mathrm{~mA}$ was coupled to an external cavity formed by the laser front facet and an end mirror $40 \mathrm{~cm}$ away.

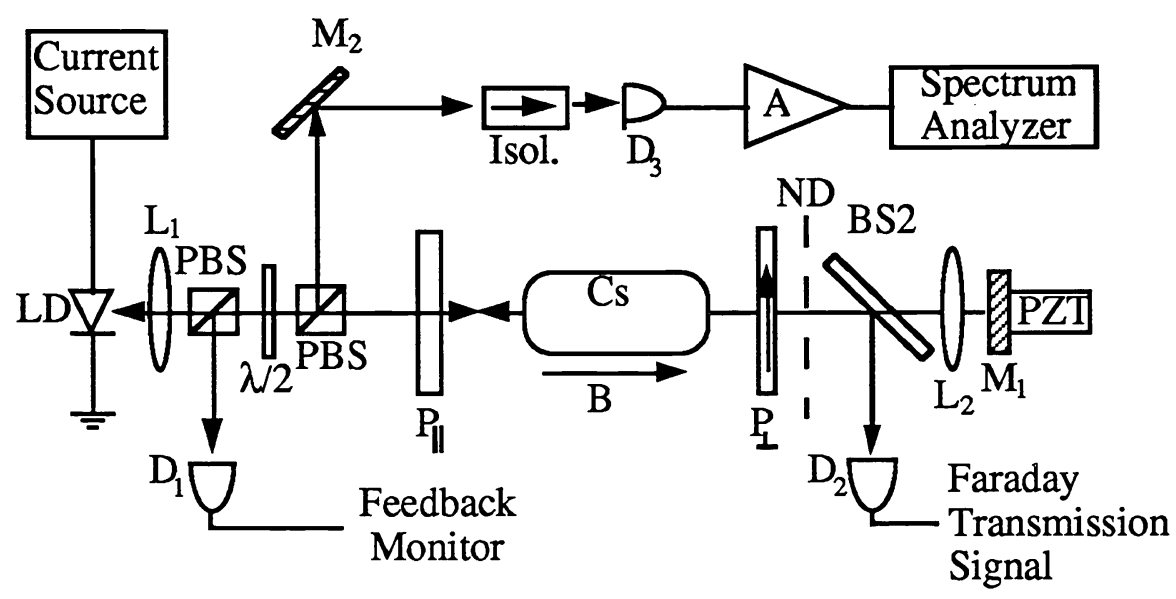

Figure 1: Experimental setup: LD, laser diode; L, lens; PBS, polarizing beamsplitter; P, polarizor; ND, neutral density filter; M, mirror; D, detector; A, amplifier; PZT, piezoelectric transducer.

The laser output beam was collimated using an AR coated microscope objective lens and then passed through a polarizing-beamsplitter/half-wave-plate/polarizing-beamsplitter combination which reflected $50 \%$ of the incident power out of the cavity for detection. The remaining transmitted field was then passed through 


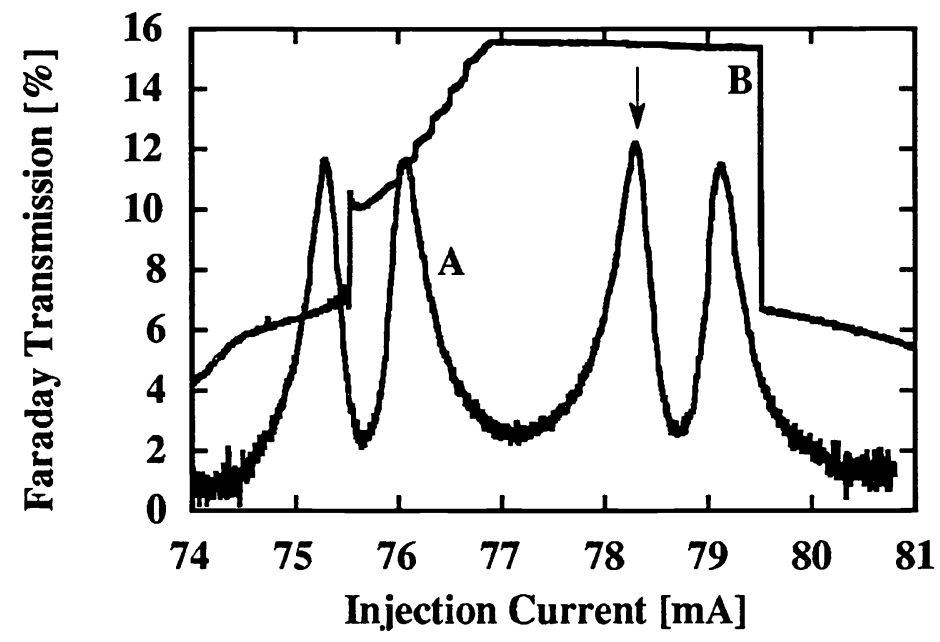

Figure 2: Faraday transmission signal obtained by scanning the laser injection current. Trace $\mathrm{A}$ is for the laser with no feedback and shows the simple Cs Faraday spectrum. Trace B is with a feedback power of $2.2 \times 10^{-2} P_{\text {out }}$.

a cell containing Cs vapor placed between two crossed polarizers. The Cs was heated to approximately $95^{\circ} \mathrm{C}$ and an axial magnetic field was applied, allowing transmission through the polarizers via Faraday rotation when the laser was tuned to the Cs D2 transition ${ }^{17}$. This transmitted portion of the beam was reflected by the end mirror back into the laser. The feedback power and phase were controlled using a neutral density filter placed in the beam path and a PZT on which the end mirror was mounted. All of the optics used in the cavity were AR coated and angled slightly to avoid spurious optical feedback.

The laser frequency could be tuned to the Cs line by carefully controlling the laser temperature and current. Long term temperature stability of better than $1 \mathrm{mK}$ was acheived using servo electronics ${ }^{18}$ and Peltier coolers attached to the laser mount. The injection current was controlled using a home-built precision current source ${ }^{19}$ with a long term stability of about $1 \mu \mathrm{A}$. With this degree of temperature and current control, highly dispersive and wavelength-selective optical feedback could be precisely applied to the laser.

The laser amplitude noise was measured using direct detection with a small-area, high speed PIN photodiode (Ortel PD050-OM). The output from the external cavity was passed through a pair of optical isolators which provided $60 \mathrm{~dB}$ of isolation. The beam was then focussed onto the detector and the resulting signal amplified with an RF amplifier and measured with an electronic spectrum analyzer.

When a magnetic field of about 500 Gauss was applied to the Cs, a feedback power of about $2 \times 10^{-2} P_{\text {out }}$ could be reflected back into the laser. Under these conditions, the laser operated in a stable, narrowlinewidth mode with a somewhat lower threshold current (due to the strong feedback), improved sidemode suppression and considerably reduced amplitude noise. If the feedback power was reduced below $10^{-2} P_{\text {out }}$, the laser linewidth broadened and the amplitude noise increased considerably indicating the onset of coherence collapse.

The locking behavior of the laser is shown in Figure 2. The laser current was scanned and the Faraday 
transmission signal was measured at D2 in Figure 1. Trace $\mathbf{A}$ is taken with the feedback from the end mirror blocked. The spectrum consists of the two Cs transitions at this wavelength $\left(6 s_{1 / 2}(F=3) \rightarrow 6 p_{3 / 2}(F=4)\right.$ and $6 s_{1 / 2}(F=4) \rightarrow 6 p_{3 / 2}(F=5)$ ), each split into a double-peaked structure due to the strong absorbtion at the line center ${ }^{17}$. Since the frequency difference between the two transitions above is $9.2 \mathrm{GHz}$, the frequency change of the laser with injection current can be deduced from trace $\mathrm{A}$ to be $3 \mathrm{GHz} / \mathrm{mA}$. Trace $\mathrm{B}$ shows the transmission spectrum with a feedback power of $2.2 \times 10^{-2} P_{\text {out }}$. The laser locked only to the strongest of the four Faraday transmission lines (indicated by an arrow in Figure 2) and had a typical locking range of around $10 \mathrm{GHz}$.

The laser LI curve, taken under feedback conditions at the peak of the scan in Figure 2, is compared with the free-running LI curve in Figure 3. It can be seen that the threshold current was reduced from $51.4 \mathrm{~mA}$ to $44.9 \mathrm{~mA}$ while the external quantum efficiency remained roughly the same. Changing the phase of the feedback did alter the output power somewhat at a fixed injection current, but at no time was the output power observed to go below that of the free-running case which would have indicated an increase in the threshold current. The laser linewidth was also measured using a delayed self-heterodyne system with a $5 \mathrm{~km}$ fiber. A typical linewidth at an injection current of $56.5 \mathrm{~mA}$ is shown in Figure 4 and was found to be reduced by a roughly factor of 2000 to $10 \mathrm{kHz}$, consistent with previously published results with this system $^{11}$.

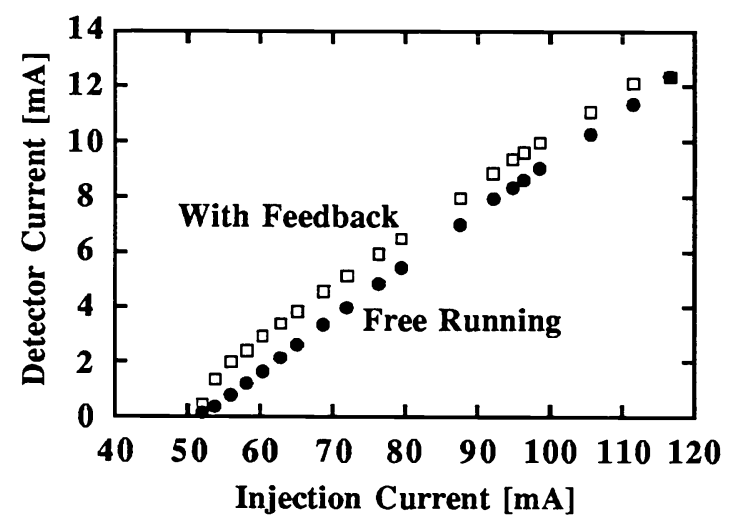

Figure 3: The LI curve of the laser both with and without feedback. The threshold current is reduced by $6.5 \mathrm{~mA}$ under feedback conditions due to the modified coupled cavity loss rate.

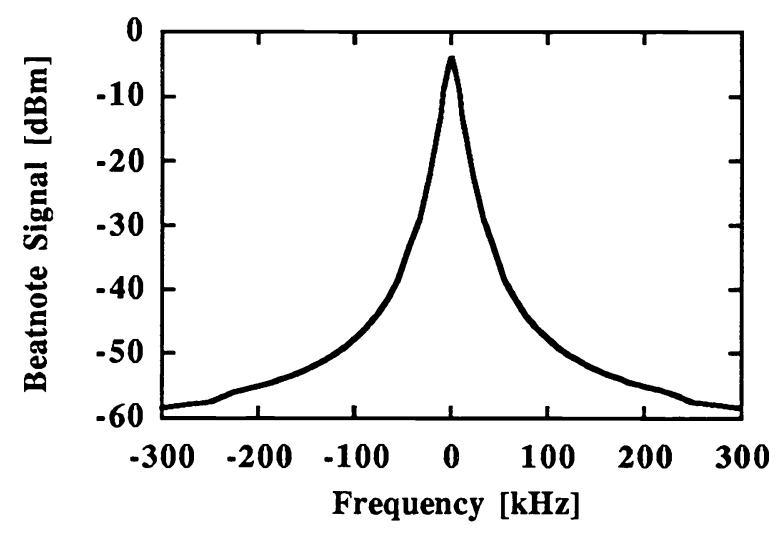

Figure 4: Linewidth of the laser under feedback conditions. The FWHM is $10 \mathrm{kHz}$

The resulting amplitude noise spectrum at two different injection currents is shown in Figure 5. Trace A shows the detector photocurrent noise power of the free-running laser while trace B shows the noise under feedback conditions at the same injection current. Since the output power was observed to increase when the 
feedback was applied, trace $\mathrm{C}$ was taken at the same output power as B (and at a higher injection current) to verify that the reduced noise was not exclusively a result of the increased output power. At low injection currents, $16 \mathrm{~dB}$ reduction in the low frequency amplitude noise is observed. In addition, the relaxation resonance peak is seen to be completely suppressed, a feature observed at all injection currents for which the measurement was made. At higher injection currents (Figure 5b), the free running laser noise approached the shot noise limit and noise reduction due to feedback was considerably smaller.

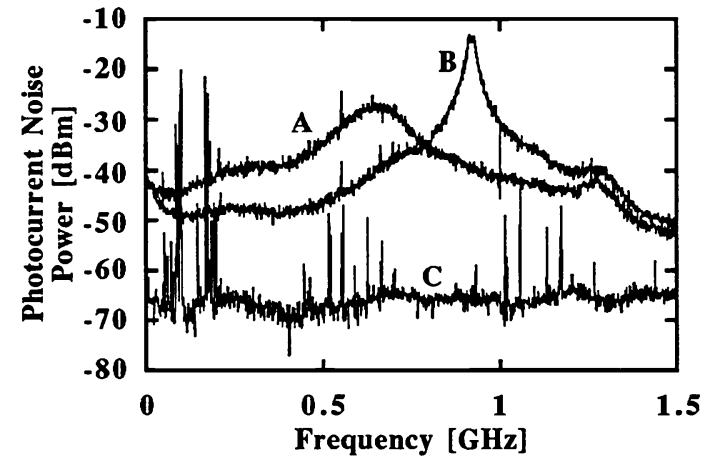

(a)

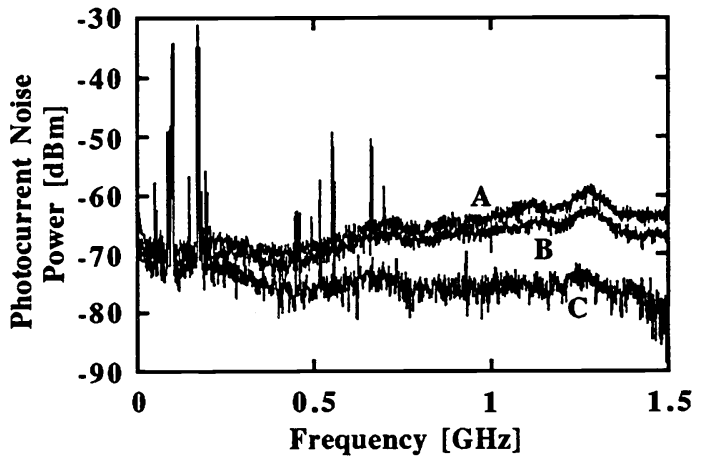

(b)

Figure 5: Photocurrent noise power of the detector indicating the laser amplitude noise: (a) $i_{L} \approx 55 \mathrm{~mA}$ and (b) $i_{L} \approx 85 \mathrm{~mA}$. Trace $\mathrm{A}$ is for the free running laser, trace $\mathrm{B}$ for the laser under feedback at the same injection current as $\mathrm{A}$ and trace $\mathrm{C}$ is for the laser free running but with the same output power as B.

Accompanying the suppression of the relaxation oscillation at low frequencies was the appearance of a strong high frequency peak in the amplitude noise spectrum. Figure 6 shows the laser amplitude noise spectrum over a frequency range of $0-20 \mathrm{GHz}$, both with and without feedback. The low frequency part of the figure shows the suppression of the relaxation oscillation at its usual frequency as described above. The high frequency part shows a strong new peak which occured under feedback conditions. This peak position was observed to vary over the range 3-22 $\mathrm{GHz}$ depending on the phase and strength of the feedback and occured over a wide range of injection currents. Although it is not well understood at present, this result is in qualitative agreement with predictions of the relaxation oscillation behaviour under feedback ${ }^{2}$ and it therefore may be an indication that the laser relaxation oscillation frequency can be increased with the application of strong optical feedback. Further investigation is required, however, to determine such characteristics as the modulation response of this coupled cavity system. 


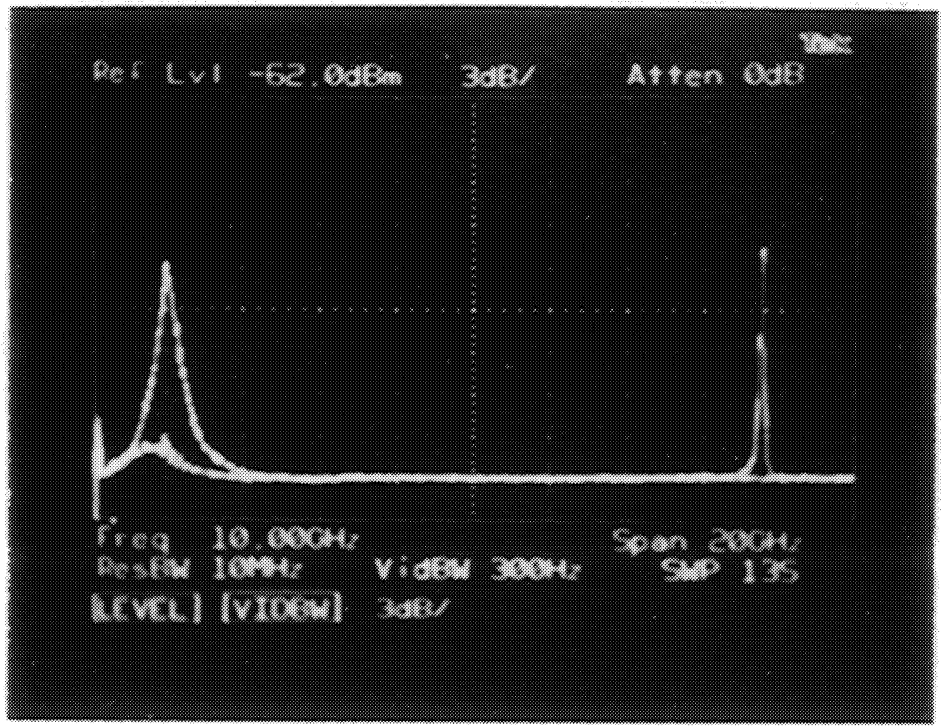

Figure 6: Amplitude noise spectrum from $0-20 \mathrm{GHz}$ of the laser both without feedback and under strong feedback conditions. The low-frequency protion shows a suppression of the relaxation oscillation while at a frequency of $18 \mathrm{GHz}$ a strong peak appears when the feedback is applied.

\section{CONCLUSION}

The effects of optical feedback on the amplitude noise spectrum of a semiconductor laser have been examined experimentally. The feedback was implemented using Faraday rotation in Cs vapor which provided a dispersive, wavelength-selective filter to which the laser was locked. It is found that with feedback powers of $\geq 10^{-2} P_{\text {out }}$, the laser oscillated in a stable, narrow linewidth mode with a somewhat reduced threshold current from the free running laser. The amplitude noise spectrum of the laser was measured under these conditions using direct detection in a photodiode and an RF spectrum analyzer. It is found that a reduction in the amplitude noise by $16 \mathrm{~dB}$ is possible at injection currents close to threshold. At higher injection currents, this reduction decreases as the laser noise approaches the shot noise limit. We have also observed a dramatic reduction of the relaxation resonance peak at its usual frequency of $\approx 1 G H z$. Accompanying this reduction is the appearance of a peak at a much higher frequency, indicating the possibility that the relaxation oscillation frequency has been considerably enhanced.

\section{References}

[1] R. Lang and K. Kobayashi, "External optical feedback effects on semiconductor injection laser properties", IEEE JQE, Vol. QE-16 (3), pp. 347-55, March 1980. 
[2] K. Vahala and A. Yariv, "Detuned loading in coupled cavity semiconductor lasers - Effects on quantum noise and dynamics", Appl. Phys. Lett., Vol. 45 (5), pp. 501-3, Sept. 1984.

[3] G. P. Agarwal and C. H. Henry, "Modulation performance of a semiconductor laser coupled to an external high-Q resonator", IEEE J. Quantum Electr., Vol. 24 (2), pp. 134-42, Feb. 1988.

[4] D. R. Hjelme, A. R. Michelson and R. G. Beausoleil, "Semiconductor laser stabilization by external optical feedback", IEEE J. Quantum Electr.,Vol. 27 (3), pp. 352-72, March 1991.

[5] H. Li and N. B. Abraham, "Analysis of the noise spectra of a laser diode with optical feedback from a high-finesse resonator", IEEE J. Quantum Electr., Vo. 25 (8), pp. 1782-93, Aug. 1989.

[6] A. Ritter and H. Haug, "Theory of laser diodes with weak optical feedback. I. Small signal analysis and side-mode spectra", J. Opt. Soc. B, Vol. 10 (1), pp. 130-44, Jan. 1993.

[7] J. S. Cohen, R. R. Drenten and B. H. Verbeek, "Effects of optical feedback on the relaxation oscillation in semiconductor lasers", J. Opt. Soc. Am. B, Vol. 10 (1), pp. 130-44, Jan. 1993.

[8] P. Spano, S. Piazzolla and M. Tamburrini, "Theory of noise in semiconductor lasers in the presence of optical feedback", IEEE J. Quantum Electr., Vol. QE-20 (4), pp. 350-7, April 1984.

[9] B. Dahmani, L. Hollberg and R. Drullinger, "Frequency stabilization of semiconductor lasers by resonant optical feedback", Opt. Lett., Vol. 12 (11), pp. 876-8, Nov. 1987.

[10] P. Laurent, A. Clairon and C. Breant, "Frequency noise analysis of optically self-locked diode lasers", IEEE J. Quantum Electr., Vol. 25 (6), pp. 1131-41, June 1989.

[11] Y. Shevy, J. Iannelli, J. Kitching and A. Yariv, "Self-quenching of the semiconductor laser linewidth below the Schawlow-Townes limit by using optical feedback", Opt. Lett., Vol. 17 (9), pp. 661-3, May 1992.

[12] A. Yariv, R. Nabiev and K. Vahala, "Self-quenching of fundamental phase and amplitude noise in semiconductor lasers with dispersive loss", Opt. Lett., Vol. 15 (23), pp. 1359-61, Dec. 1990.

[13] H. Sato, T Fujita and K. Fujito, "Intensity fluctuations in semiconductor lasers coupled to external cavity", IEEE J. Quantum Electr., Vol. QE-21 (1), pp. 46-51, Jan. 1985.

[14] H. Haug, "Quantum mechanical rate equations for semiconductor lasers", Phys. Rev., Vol. 184 (2), pp. 338-48, Aug. 1969.

[15] Y. Yamamoto, S. Machida and O. Nilsson, "Amplitude squeezing in a pump-suppressed laser oscillator", Phys. Rev. A, Vol. 34 (5), 4025-42, Nov. 1986.

[16] R. Nabiev, Y. Popov and A. Yariv, "Semiconductor laser with dispersive loss: quantum noises and amplitude squeezing”, J. Phys. III, Vol.2 (9), pp. 1605-14, Sept. 1992.

[17] J. Menders, K. Benson, S. H. Bloom, C. S. Liu and E. Korevaar, "Ultranarrow line filtering using a Cs Faraday filter at 852 nm", Opt. Lett., Vol. 16 (11), pp. 846-8, June 1991.

[18] C. C. Bradley, J. Chen and R. Hulet, "Instrumentation for the stable operation of laser diodes", Rev. Sci. Instrum., Vol. 61 (8), pp. 2097-2101, Aug.1990. 
[19] K. G. Libbrecht and J. L. Hall, "A low-noise diode laser current controller", Rev. Sci. Instrum., Vol.64 (8), pp. 2133-5, Aug. 1993.

[20] D. Lenstra, B. H. Verbeek and A. J. den Boef, "Coherence collapse in single-mode semiconductor lasers due to optical feedback", IEEE J. Quantum Electr., Vol. QE-21, pp. 674-9, June 1985. 